\title{
Serotonin synthesis and metabolism-related molecules in a human prostate cancer cell line
}

\author{
TOSHIAKI SHINKA ${ }^{1}$, DAI ONODERA ${ }^{3}$, TETSUJI TANAKA ${ }^{2}$, NORIAKI SHOJI ${ }^{3}$, \\ TADAAKI MIYAZAKI ${ }^{4}$, TETSUYA MORIUCHI ${ }^{5}$ and TAKAHIRO FUKUMOTO ${ }^{6}$
}

\begin{abstract}
Departments of ${ }^{1}$ Urology, and ${ }^{2}$ Obstetrics and Gynecology, Wakayama Medical University, Wakayama 641-0012;
${ }^{3}$ Department of Oral Medicine and Surgery, Division of Oral Diagnosis, Tohoku University Graduate School of Dentistry, Aoba-ku 980-8575; ${ }^{4}$ Department of Bioresources, Hokkaido University Research Center for Zoonosis Control, Sapporo 001-0020; ${ }^{5}$ Division of Cancer-Related Genes, and ${ }^{6}$ Research Center for Infection-Associated Cancer, Institute for Genetic Medicine, Hokkaido University, Sapporo 060-0815, Japan
\end{abstract}

Received September 9, 2010; Accepted December 27, 2010

DOI: $10.3892 / \mathrm{ol} .2011 .244$

\begin{abstract}
Prostate cancer is one of the most common tumors in males and its incidence is steadily increasing worldwide. Serotonin or 5-hydroxytryptamine (5-HT) is a well-known neurotransmitter that mediates a wide variety of physiological effects. An increase in the number of 5-HT-releasing neuroendocrine (NE) cells has been correlated with tumor progression. However, it is particularly unclear whether released 5-HT or the release of 5-HT has a role in tumor cell growth. We hypothesized that 5-HT synthesis and metabolism in NE cells regulate the growth of prostate cancer cells. In the present study, 5-HT was found to play a role as a cell growth factor in prostate cancer cells. Moreover, the pharmacological inhibition of 5-HT synthesis and metabolism interrupted the growth of prostate cancer cells. To confirm the existence of 5-HT in prostate cancer cells, we performed ELISA, HPLC, RT-PCR and immunohistochemical analyses. A high expression of tryptophan hydroxylase (TPH-1), dopa decarboxylase (DDC) and monoamine oxidase A (MAO-A) was noted in the prostate cancer cells when compared with normal prostate cells. Previous studies showed that 5-HT stimulated the proliferation of prostate cancer cells mediated by 5-HT receptors 5-HTR1A and R1B. However, cell proliferation was significantly inhibited when siRNA for both DDC and TPH-1 was transfected to the cells. Consequently, we propose that the secretion system of prostate NE cells capable of 5-HT synthesis and metabolism plays a significant role in prostate tumor generation and progression. These findings provide crucial clues for the development of potential pharmacotherapeutics to slow prostate tumor progression.
\end{abstract}

Correspondence to: Dr Takahiro Fukumoto, Research Center for Infection-Associated Cancer, Institute for Genetic Medicine, Hokkaido University, Kita-15, Nishi-7, Kita-ku, Sapporo 060-0815, Japan

E-mail: takataka@igm.hokudai.ac.jp

Key words: prostate cancer, serotonin, dopa decarboxylase

\section{Introduction}

Prostate cancer is the most frequently diagnosed invasive cancer and the leading cause of cancer-related death among males in a number of countries (1). Mounting evidence indicates that neuroendocrine (NE) cells play a role in prostatic disease. NE cells populate both normal and malignant prostate tissues (2), and can synthesize, store and release growth factors, as well as biogenic amines, serotonin 5-hydroxytryptamine (5-HT), dopamine and other neurotransmitter-related substances (3-5). An increase in the number of NE cells is related to tumor progression (6). However, whether an increase in NE cell secretory products as well as their synthesis and metabolism contribute to tumor progression remains to be determined.

Serotonin is a well-known neurotransmitter which exhibits multiple non-neural functions involved in essential hypertension (7), early embryogenesis (8), follicle maturation (9) and behavior (10). Serotonin acts as a growth factor in various types of non-tumor cells and has been associated with oncogenes $(11,12)$. The essential amino acid tryptophan is the precursor of 5-HT, and is synthesized in two steps catalyzed by the enzymes, tryptophan hydroxylase (TPH) and dopa decarboxylase (DDC). The major metabolic product of 5-HT is 5-hydroxyindoleacetic acid (5-HTIAA) via degradation by monoamine oxidase A (MAO-A) (Fig. 1A). TPH is the rate-limiting enzyme in 5-HT synthesis and exists in the forms of TPH-1 and TPH-2. TPH-1 is generally found in the pineal body and gut, and TPH-2 is selectively expressed in brain. DDC is known as a key molecule for neuronal disease (13). Findings of a recent study indicated that DDC is a novel co-activator of the androgen receptor (AR) in prostate tissues (14).

\section{Materials and methods}

Reagents. Serotonin and all drugs were purchased from Sigma-Aldrich (St. Louis, MO, USA). Reagents were dissolved in sterile distilled water or dimethyl sulfoxide. 
In vitro proliferation assay. $\mathrm{LNCaP}$ and $\mathrm{PC}-3$ cells were seeded in a 96-well plate and cultured in serum containing media at $37^{\circ} \mathrm{C}$. After $24 \mathrm{~h}$, the cells were washed with phosphate-buffered saline (PBS) twice, and then replaced with the reagents (serotonin and antagonists) containing culture media [fetal bovine serum (FBS; +FBS or -FBS)]. Cell proliferation was assessed, and changes in cell number were quantified using an Alamar blue assay (Invitrogen) and trypan blue measurement $96 \mathrm{~h}$ after adding the reagents. Briefly, after adding $10 \mu \mathrm{l}$ Alamar blue reagent (Invitrogen) to the well (100 $\mu \mathrm{l}$ media), the 96-well plates were incubated at room temperature for $1 \mathrm{~h}$. The optical density of the cells was read at $570 \mathrm{~nm}$ using a spectrophotometric plate reader. To confirm the Alamar blue assay, the cell numbers were measured using trypan blue staining. Each experiment was repeated on five separate occasions, each time using a quadruple sample. Cell viability is expressed as a percentage of the optical density of the control, defined as $100 \%$. Data were analyzed, and the statistical significance was accepted at $\mathrm{P}<0.05$.

Serotonin ELISA assay. The serotonin ELISA kit was purchased from IBL, Germany. Each cell line was homogenized at $4^{\circ} \mathrm{C}$ in RIPA buffer $[50 \mathrm{mM}$ Tris- $\mathrm{HCl}$ (pH.8.0), $150 \mathrm{mM} \mathrm{NaCl}, 1 \% \mathrm{NP}-40,0.5 \%$ DOC, $0.1 \%$ SDS, $50 \mathrm{mM} \mathrm{NaF}$, $1 \mathrm{mM}$ EDTA, $10 \mu \mathrm{g} / \mathrm{ml}$ leupeptin, $10 \mathrm{KIU} / \mathrm{ml}$ aprotinin, $1 \mathrm{mM}$ PMSF, $1 \mathrm{mM}$ DTT] and adjusted to $1 \mathrm{mg} / \mathrm{ml}$ using the Protein Assay kit (BioRad, Japan). The ELISA and protein assays were performed according to the manufacturer's instructions and were repeated 3 times each. Data were analyzed, and the statistical significance was accepted at $\mathrm{P}<0.05$.

Serotonin measurement using high performance liquid chromatography. Each cell line was homogenized in $0.2 \mathrm{~mol} / 1$ ice-cold perchloric acid, and the homogenate was cooled in ice for $0.5 \mathrm{~h}$ for deproteinization. The homogenate was centrifuged at 20,000 x g for $10 \mathrm{~min}$ at $4^{\circ} \mathrm{C}$. The sample was then filtered through a $0.45-\mu \mathrm{m}$ filter (Millipore, USA) at $20,000 \mathrm{xg}$ for $20 \mathrm{~min}$ at $4^{\circ} \mathrm{C}$. The $30-\mu l$ filtrate was applied to a high performance liquid chromatography (HPLC) system (Eicom, Kyoto, Japan) with a 150x2.1 mm octadecyl silane column (SC-50DS, Eicom) and electrochemical detector (ECD-300, Eicom) at an applied potential of $+700 \mathrm{mV}$ versus an $\mathrm{Ag} / \mathrm{AgCl}$ reference analytical electrode. Changes in electric current (nA) were recorded by computer using an interface system (Power Chrom ver. 2.3.2.J). The mobile phase was composed of aceto-citric acid buffer $(0.1 \mathrm{M})$, methanol, sodium-1-octane sulfonate $(0.46 \mathrm{M})$ and disodium ethylenediaminetetraacetic acid $(0.015 \mathrm{M})(830: 170: 1.9: 1)$ at a flow rate of $0.2 \mathrm{ml} / \mathrm{min}$. The concentrations of 5-hydroxytryptophan, serotonin and 5-HTIAA were determined, and the cell levels were calculated.

Serotonin detection using immunohistochemistry. Cells were cultured on 8-well chamber slides, fixed in 4\% paraformaldehyde (Sigma-Aldrich) and permeabilized in 98\% ice-cold ethanol. Donkey serum (10\% v/v) was used to block the nonspecific binding of antibodies. The slide was then incubated at room temperature for $1 \mathrm{~h}$ in the primary antibodies. A rabbit polyclonal antibody against serotonin was used (Sigma) (1:2000). The slides were washed and incubated with Alexa Fluor 594 goat anti-rabbit IgG (Invitrogen) (1:1000) at room temperature for $1 \mathrm{~h}$, and then washed and incubated with DAPI antibody for DAPI staining.

$R T$-PCR analysis. RNA was isolated from the LNCaP, PC-3 and PrEC cell lines using TRIzol reagent (Invitrogen) according to the manufacturer's instructions. cDNA synthesis was performed using SuperScript II (Invitrogen) for RT-PCR. Amplification of the human TPH-1 (Gene Bank: NM_004179), human TPH-2 (Gene Bank: NM_173353), human DDC (Gene Bank: BC008366), human AR (Gene Bank: L29496), human MAO-A (Gene Bank: NM_000240), human PSA (Gene Bank: DQ893851), human androgen receptor (AR) (Gene Bank: L29496), and human GAPDH (Gene Bank: NM_002046) was conducted using specific primers and PCR conditions. The primers used were: Human TPH-1, forward: 5'-ATGATTGAAG ACAATAAGGAG and reverse: 5'-AAGTTTTTGAGATACT CTCTG; human TPH-2,forward: 5'-ATGCAGCCAGCAATGA TGATGT and reverse: 5'-ACATCCTCTAGCTCTTCTTCCT; human DDC, forward: 5'-ATGAACGCAAGTGAATTCCGA AGG and reverse: 5'-GCCTTTGGTAGTTCCAGCATCTTC; human MAO-A, forward: 5'-AGTATCGCGGGCCACATGTT and reverse: 5'-ACCGCCTAGCAGTCTTTGTC; human AR, forward: 5'-ATGCAACTCCTTCAGCAACAGC and reverse: 5'-GGACTTGTAGAGAGACAGGGTA; human PSA, forward: 5'-ATGTGGGTCCCGGTTGTCTT and reverse: 5'-GTCCA TGACCTTCACAGCATCC, and human GAPDH, forward: 5'-GCCTGGTCACCAGGGCTGCTTT and reverse: 5'-GCC AGGGGTGCTAAGCAGTTGG. The PCR conditions were: initial denaturation of $94^{\circ} \mathrm{C}$ for $5 \mathrm{~min}$ followed by 20 cycles at $94^{\circ} \mathrm{C}$ for $30 \mathrm{sec}$, and $51^{\circ} \mathrm{C}$ (for TPH-1), $52^{\circ} \mathrm{C}$ (for TPH-2), $56^{\circ} \mathrm{C}$ (for DDC), $57^{\circ} \mathrm{C}$ (for MAO-A), $54^{\circ} \mathrm{C}$ (for $\mathrm{AR}$ ), $63^{\circ} \mathrm{C}$ (for PSA), or $65^{\circ} \mathrm{C}$ (for GAPDH) for $20 \mathrm{sec}$, and a final extension of $72^{\circ} \mathrm{C}$ for $15 \mathrm{sec}$. Negative controls with no RT product were routinely performed (data not shown).

Small interfering RNA transfection. siRNAs were purchased for the targeting of human DDC and TPH-1 (Santa Cruz Biotechnology, Santa Cruz, CA, USA) which were transfected to LNCaP cells following the manufacturer's instructions. Briefly, cells were seeded in a 6 -well plate at $1 \times 10^{5}$ cells/well in antibiotic-free media containing 10\% FBS. Following a 20-h incubation, the siRNA solution was added to the well, and incubation was carried out at $37^{\circ} \mathrm{C}$ for $5 \mathrm{~h}$. Cells were washed 3 times with PBS, and the media were replaced with fresh media supplemented with antibiotics (penicillin/streptomycin) and 5-HT (final volume of $10 \mu \mathrm{M}$ ). Cell proliferation was assessed, and changes in the cell numbers were quantified using the Alamar blue assay and trypan blue measurement after 3, 5 and 7 days of incubation.

\section{Results}

Serotonin 5-HT acts as a cell growth factor, as previously reported (9). As shown in Fig. 1B, 5-HT promoted the proliferation of a prostate cancer cell line, $\mathrm{LNCaP}$, in a dose-dependent manner. The PC-3 and DU145 cell lines also provided similar results (data not shown). To further confirm this activity, the same experiments were conducted again using serum-free media (red bar, Fig. 1B). 5-HT was found in serum and was circulated in the entire body for the maintenance of 
A

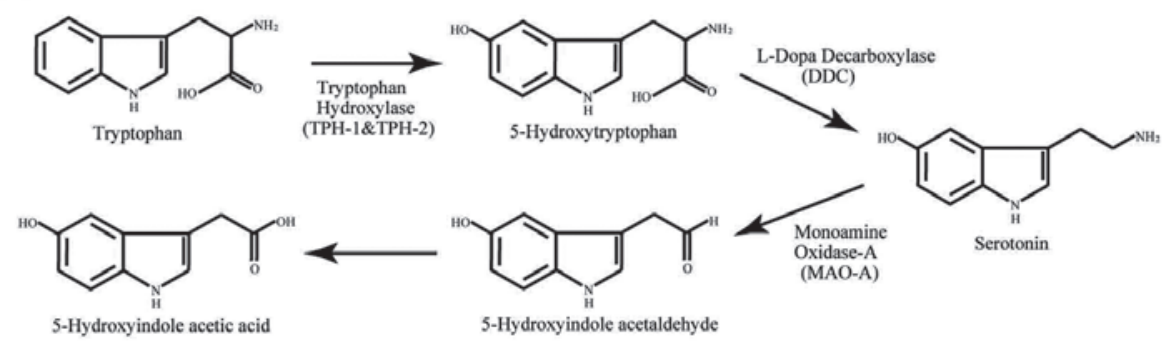

C

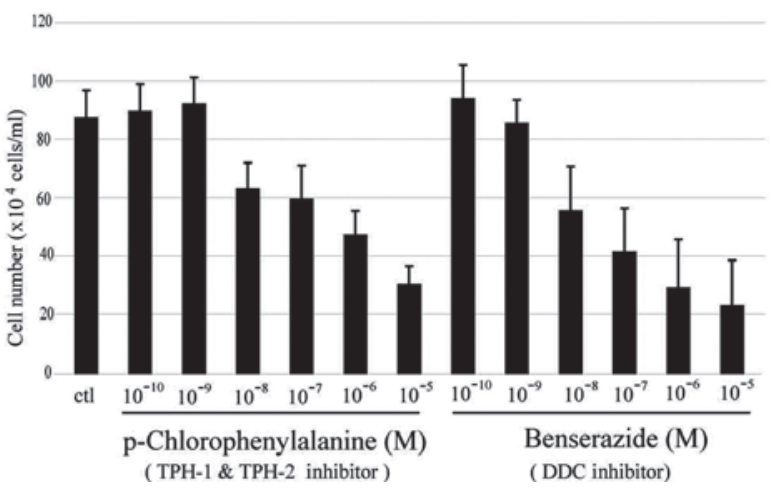

B

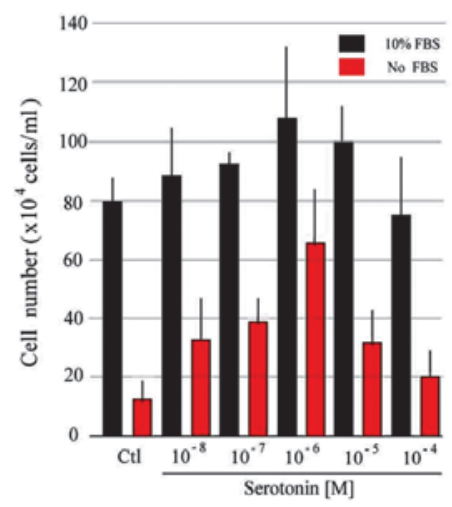

D

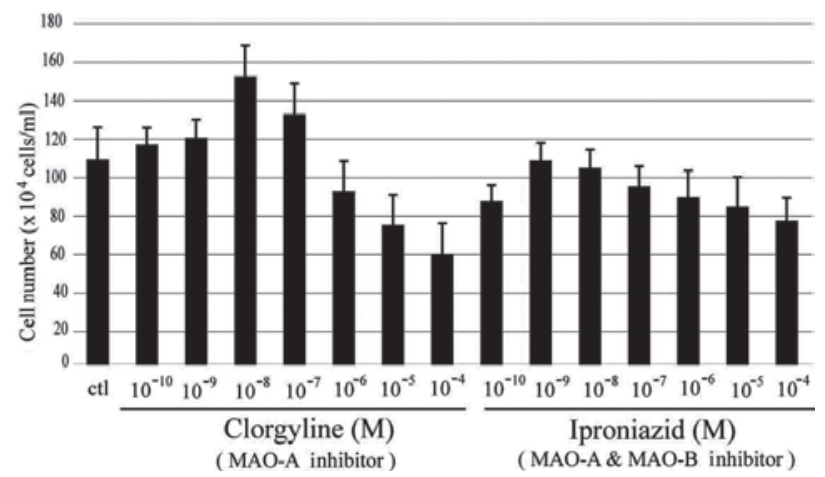

Figure 1. The serotonin synthesis and metabolism pathway and components affect cell proliferation. (A) Chemical structural pathway of the serotonin synthesis and metabolism system. (B) 5-HT significantly stimulated the growth of LNCaP cells in a dose-dependent manner (Black bar, +FBS; red bar, -FBS). (C) Antagonists of serotonin synthesis enzymes (p-chlorophenylalanine and benserazide) inhibited LNCaP cell growth by $50 \%$ at a concentration of $10 \mu \mathrm{M}$ compared to the control at $72 \mathrm{~h}$. (D) At a concentration of $0.01 \mu \mathrm{M}$, monoamine oxidase A (MAO-A)-specific antagonist (clorgyline) induced the cell growth effect by $30 \%$, while treatment with a higher concentration showed $40 \%$ inhibition of cell proliferation compared to the control cells. Non-selective MAO antagonist (iproniazid) had no significant inhibitory effect on cell growth.

homeostasis (15). The role of 5-HT as a cell growth factor was indicated when the results of our experiments were compared with those using serum-containing media. In LNCaP cells, inhibitors of 5-HT synthesis, p-chlorophenylalanine (Sigma, C8655) for TPHs (Fig. 1C) and benserazide (Sigma, B7283) for DDC (Fig. 1C) had an almost $50 \%$ inhibitory effect on cell proliferation at a concentration of $10 \mu \mathrm{M}$ at $72 \mathrm{~h}$ of culture incubation compared to the control cells. Other antagonists, including fenfluramine for tryptophan hydroxylase (TPH) and carbidopa for dopa decarboxylase (DDC), showed a similar inhibitory effect on the three cell lines (data not shown). The effect of the irreversible inhibitor of monoamine oxidase A (MAO-A) (clorgyline, Sigma, M3778) on LNCaP cells was also investigated (Fig. 1D). In the presence of $0.01 \mu \mathrm{M}$ clorgyline, cell growth was increased by $30 \%$ when compared to the controls, although a higher concentration of clorgyline resulted in $40 \%$ inhibition of cell proliferation when compared to the controls. The non-selective MAO inhibitor, iproniazid (Sigma, I7627) exhibited no significant inhibitory effect on cell growth (Fig. 1D). Each proliferation assay in Fig. 1 was repeated on five separate occasions. The results strongly indicate that the 5-HT synthesis and metabolism system plays a regulatory role in the proliferation of these prostate cell lines.

To investigate the expression of 5-HT, TPH-1, DDC and MAO-A in normal prostate cells (PrEC, Takara, C2555) and prostate cancer cell lines (LNCaP and PC-3), we used a combination of immunohistochemical, ELISA, and HPLC analyses for 5-HT, and RT-PCR for the remaining molecules. We first analyzed for 5-HT synthesis and mRNA expression of the metabolic components by RT-PCR using specific primers in PrEC, LNCaP and PC-3 cells. Moreover, we analyzed prostate-specific antigen (PSA) and AR, which were already characterized in the prostate cell lines (Fig. 2A). The expression of DDC in the PC-3 cells was 3 times that of PrEC cells but no significant difference was noted in the DDC expression between the LNCaP and PrEC cell lines. On the other hand, a high MAO-A expression correlated with that in the LNCaP cells. PC-3 cells exhibited the lowest expression of TPH-1 when compared to the three cell lines. These data indicate that 5-HT synthesis and metabolic components are expressed in prostate cell lines. We then investigated whether differences in the expression of each gene in the PrEC, LNCaP and PC-3 cells correlated with the production of 5-HT in these cell lines. Serotonin immunoreactivity was detected in these cells using an anti5-HT Ab, and whole cell conditions were confirmed by DAPI-staining (Fig. 2B, inset panels). Serotonin content as measured by ELISA showed different values for the three cell lines (Fig. 2B). Although, a small difference in 5-HT content between the normal prostate and prostate cancer cell lines was anticipated, PC-3 cells exhibited almost 3 times the amount of 5-HT compared to the LNCaP cell line which consisted of half of the 5-HT content of the PrEC cells. The contents of the 5-HT precursor (5-HTP), 5-HT, and 5-HIAA were also measured using HPLC (Fig. 2C). The results correlated to a similar extent with the gene expression levels. 
A

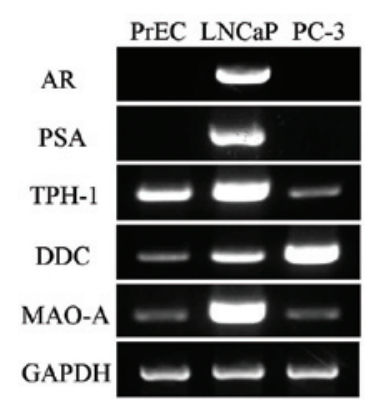

B

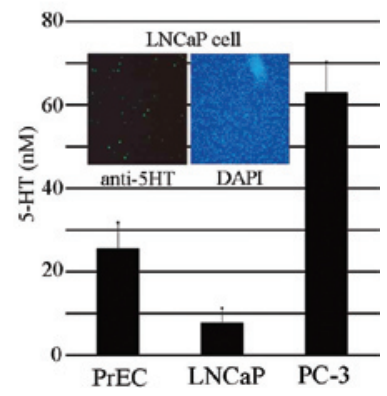

C

\begin{tabular}{llcc} 
& PrEC & LNCaP & PC-3 \\
\hline 5-HTP (nM) & $15.9 \pm 8$ & $10.3 \pm 2$ & $56.9 \pm 8$ \\
5-HT (nM) & $29.6 \pm 11$ & $9.2 \pm 3$ & $68.8 \pm 16$ \\
5-HIAA (nM) & $37.8 \pm 21$ & $42.2 \pm 8$ & $103.8 \pm 46$ \\
\hline
\end{tabular}

Significant difference compared to the control at $\mathrm{P}<0.05$.

Figure 2. The serotonin synthesis and metabolism system plays a role in prostate cancer cells. (A) Total RNA was isolated from PrEC, LNCaP and PC-3 cells. Following reverse transcription, PCR was performed using specific primers for the components. (B) ELISA analysis of the serotonin content in both normal prostate and cancer cell lines. PC-3 cells had a high serotonin content, whereas LNCaP cells had a low serotonin content as compared to the normal prostate cell line. Inset, immunohistochemical analysis with both anti-5-HT Ab and DAPI staining in LNCaP cells. (C) HPLC analysis for 5-HTP, 5-HT and 5-HIAA contents in prostate-related cell lines.

A

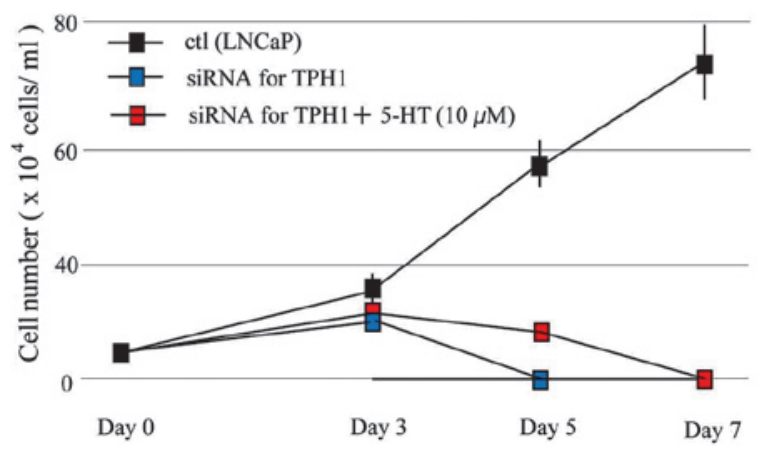

B

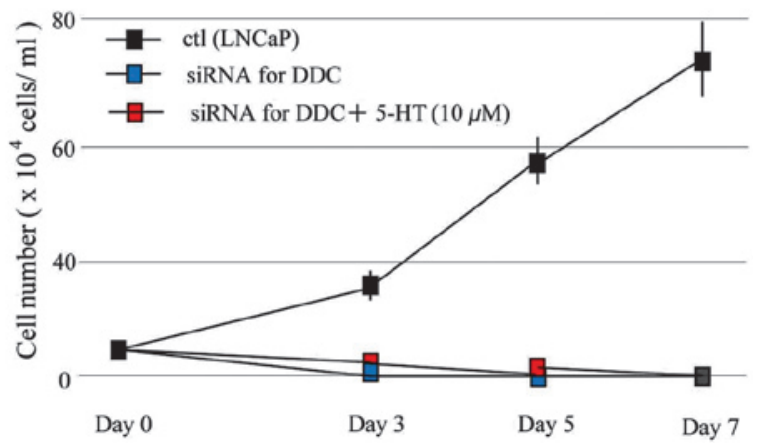

Figure 3. Dopa decarboxylase (DDC) and tryptophan hydroxylase (TPH-1) siRNA expression inhibits cell proliferation. LNCaP cells were cultured in RPMI1640 with $10 \%$ FBS. After siRNA was transfected to the cell lines (A, TPH-1 target; B, DDC target), the cells were cultured with 5-HT (10 $\mu$ M) or without 5-HT for 7 days. (Black box, control; blue box, siRNA only; red box; siRNA + 5-HT)

Thus, serotonin was synthesized and metabolized in each cell line and regulated the identity of cells by its release.

The effect of signaling via 5-HT receptors is considered to have an essential role in the effects of cell proliferation of 5-HT (16). However, our results indicate that both the synthesis and release of 5-HT in cells plays a role in cell proliferation. Thus, these effects were investigated using siRNA targeted to DDC and TPH-1 in LNCaP cells (Fig. 3). Cells treated with each siRNA inhibited growth almost completely, and the addition of 5-HT did not induce cell proliferation. These results show that the serotonin synthesis and metabolism system has an essential role in cell growth.

\section{Discussion}

This is the first study to show that serotonin or 5-HT synthesis and its release are involved in prostate cancer cell lines. The primary hypothesis regarding serotonin involvement in prostate cancer was proposed by Lembeck in 1953 (17). On the other hand, a more general hypothesis termed the APUD (amine precursor uptake and decarboxylation) cell concept (18) has been proposed based on the synthesis and metabolism system. Regarding prostate cancer generation, much evidence from clinical research can be elucidated in light of the APUD cell concept.

Our results are also in accordance with the APUD cell concept. 5-HT is found in both normal prostate and cancer cell lines, and the system of 5-HT synthesis and its release is functional. Thus, 5-HT is essential for normal cell proliferation. In the event of deviation to the 5-HT synthesis process, the initial step of tumor progression may occur since the gene knockdown of dopa decarboxylase (DDC) and tryptophan hydroxylase (TPH-1), using siRNA in the cell lines, was found to inhibit cell proliferation. Moreover, additional 5-HT stimulation to the gene did not affect cell proliferation in the cell lines. Evidence indicates that DDC binds directly with AR, which has an essentia0l role in prostate tumor generation and prostate cancer progression (17). LNCaP cells exhibit AR-dependent cell growth as compared to PC-3 cells. This fact indicates that DDC is more essential than AR in prostate cancer progression in that DDC-knockout mouse show a lethal phenotype. No literature regarding prostate in the mouse is currently available, but DDC conditional mouse targeted to prostate affects prostate tissues (unpublished data). The 5-HT synthesis process, mainly involving DDC, may regulate prostate cell proliferation directly. Thus, the development of specific DDC inhibitors 
are crucial for the future clinical assessment of DDC as an effective cancer target.

\section{Acknowledgements}

This study was supported by grants-in-aid from the Ministry of Education, Science and Culture of Japan.

\section{References}

1. Chin SN, Wang L, Moore M and Sridhar SS: A review of the patterns of docetaxel use for hormone-resistant prostate cancer at the Princess Margaret Hospital. Curr Oncol 17: 24-29, 2010.

2. Sandberg AA: Endocrine control and physiology of the prostate Prostate 1: 169-184, 1980.

3. Abrahamsson PA: Prostate cancer and active surveillance. Front Radiat Ther Oncol 41: 1-6, 2008.

4. Hansson J and Abrahamsson PA: Neuroendocrine differentiation in prostatic carcinoma. Scand J Urol Nephrol Suppl 212: 28-36, 2003.

5. Seuwen K and Pouyssegur J: Serotonin as a growth factor. Biochem Pharmacol 39: 958-990, 1990.

6. Waguespack SG, Rich T, Grubbs E, Ying AK, Perrier ND, AyalaRamirez $\mathrm{M}$ and Jimenez C: A current review of the etiology, diagnosis, and treatment of pediatric pheochromocytoma and paraganglioma. J Clin Endocrinol Metab 95: 2023-2037, 2010.

7. Rapport MM, Green AA and Page IH: Serum vasoconstrictor (serotonin) IV: Isolation and characterization. J Biol Chem 176 $1243-1251,1948$.

8. Fukumoto T, Kema PI and Levin M: Serotonin signaling is a very early step in patterning of the left-right axis in chick and frog embryos. Curr Biol 15: 794-803, 2005.
9. Buznikov GA, Lambert HW and Lauder JM: Serotonin and serotonin-like substances as regulators of early embryogenesis and morphogenesis. Cell Tissue Res 305: 177-186, 2001.

10. Marino $\mathbf{J}$ and Caballero $\mathrm{J}$ : Iloperidone for the treatment of schizophrenia. Ann Pharmacother 44: 863-870, 2010.

11. Julius D, Livelli TJ, Jessel TM and Axel R: Ectopic expression of serotonin 1c receptor and triggering of malignant transformation. Science 244: 1057-1062, 1989.

12. Siddiqui EJ, Thompson CS, Mikhailidis DP and Mumtaz FH: The role of serotonin in tumour growth (Review). Oncol Rep 14 1593-1597, 2005.

13. Feng LR and Maguire-Zeiss KA: Gene therapy in Parkingson's disease: rationale and current status. CNS Drugs 24: 177-192, 2010.

14. Margiotti K, Wafa LA, Cheng H, Novelli G, Nelson CC and Rennie PS: Androgen-regulated genes differentially modulated by the androgen receptor coactivator L-dopa decarboxylase in human prostate cancer cells. Mol Cancer 6: 38-50, 2007.

15 Richard DM, Dawes MA, Mathias CW, Acheson A, HillKapturczak N and Dougherty DM: L-tryptophan: basic metabolic functions, behavioral research and therapeutic indications. Int J Tryptophan Res 2: 45-60, 2009.

16. Siddiqui EJ, Shabbir M, Mikhailidis DP, Thompson CS and Mumtaz FH: The role of serotonin (5-hydroxytryptamine 1A and $1 \mathrm{~B}$ ) receptors in prostate cancer cell proliferation. J Urol 176: 1648-1653, 2006.

17. Lembeck F: 5-Hydroxytryptamine in a carcinoid tumor. Nature 172: 910-911, 1953

18. Pearse AG: The diffuse neuroendocrine system and the APUD concept: related 'endocrine' peptides in brain, intestine, pituitary, placenta, and anuran cutaneous glands. Med Biol 55: 115-125, 1977. 